\title{
OPEN Analysis of potential regulatory LncRNAs and CircRNAs in the oxidative myofiber and glycolytic myofiber of chickens
}

\author{
Xiaojun Ju' ${ }^{1}$ Yifan Liu ${ }^{2}$, Yanju Shan², Gaige Ji², Ming Zhang ${ }^{2}$, Yunjie Tu², Jianmin Zou ${ }^{2}$, \\ Xingyong Chen ${ }^{1}$, Zhaoyu Geng ${ }^{1 \bowtie}$ \& Jingting Shu ${ }^{2 \bowtie}$
}

SART and PMM are mainly composed of oxidative myofibers and glycolytic myofibers, respectively, and myofiber types profoundly influence postnatal muscle growth and meat quality. SART and PMM are composed of IncRNAs and circRNAs that participate in myofiber type regulation. To elucidate the regulatory mechanism of myofiber type, IncRNA and circRNA sequencing was used to systematically compare the transcriptomes of the SART and PMM of Chinese female Qingyuan partridge chickens at their marketing age. The luminance value $\left(L^{*}\right)$, redness value $(a *)$, average diameter, cross-sectional area, and density difference between the PMM and SART were significant $(p<0.05)$. ATPase staining results showed that PMMs were all darkly stained and belonged to the glycolytic type, and the proportion of oxidative myofibers in SART was $81.7 \%$. A total of 5420 IncRNAs were identified, of which 365 were differentially expressed in the SART compared with the PMM $(p<0.05)$. The cis-regulatory analysis identified target genes that were enriched for specific GO terms and KEGG pathways $(p<0.05)$, including striated muscle cell differentiation, regulation of cell proliferation, regulation of muscle cell differentiation, myoblast differentiation, regulation of myoblast differentiation, and MAPK signaling pathway. Pathways and coexpression network analyses suggested that XR_003077811.1,XR_003072304.1,XR_001465942.2, XR_001465741.2, XR_001470487.1, XR_003077673.1 and XR_003074785.1 played important roles in regulating oxidative myofibers by TBX3, OKI, MYBPC1, CALM2, and PPARGC1A expression. A total of 10487 circRNAs were identified, of which 305 circRNAs were differentially expressed in the SART compared with the PMM $(p<0.05)$. Functional enrichment analysis showed that differentially expressed circRNAs were involved in host gene expression and were enriched in the AMPK, calcium signaling pathway, FoxO signaling pathway, p53 signaling pathway, and cellular senescence. Novel_circ_004282 and novel_circ_002121 played important roles in regulating oxidative myofibers by PPP3CA and NFATC1 expression. Using IncRNA-miRNA/circRNA-miRNA integrated analysis, we identified many candidate interaction networks that might affect muscle fiber performance. Important IncRNA-miRNAmRNA networks, such as IncRNA-XR_003074785.1/miR-193-3p/PPARGC1A, regulate oxidative myofibers. This study reveals that IncXR_003077811.1, IncXR_003072304.1, IncXR_001465942.2, IncXR_001465741.2, IncXR_001470487.1, IncXR_003077673.1, XR_003074785.1, novel_circ_004282 and novel_circ_002121 might regulate oxidative myofibers. The IncRNA-XR_003074785.1/miR-193-3p/ PPARGC1A pathway might regulate oxidative myofibers. All these findings provide rich resources for further in-depth research on the regulatory mechanism of IncRNAs and circRNAs in myofibers.

Myofiber is the basic constituent unit of skeletal muscle. Different types of myofibers give skeletal muscle-specific physiological characteristics and functions ${ }^{1,2}$. It is generally believed that the total number of myofibers remains unchanged after birth. However, these myofibers are dynamic structures capable of changing their phenotype during the growth process ${ }^{3}$. In chickens, myofibers can be divided into oxidative (type I and IIa, red) and glycolytic (type IIb, white) types ${ }^{4}$. The meat quality of muscle with a high proportion of oxidative myofibers is significantly

${ }^{1}$ College of Animal Science and Technology, Anhui Agricultural University, Hefei 230036, Anhui, China. ${ }^{2}$ Key Laboratory for Poultry Genetics and Breeding of Jiangsu Province, Poultry Institute, Chinese Academy of Agricultural Sciences, Yangzhou 225125, Jiangsu, China.『email: gzy@ahau.edu.cn; shujingting@163.com 
better than that of muscle with a high proportion of glycolytic myofibres ${ }^{5}$. Compared to glycolytic myofibers, oxidative myofibers are characterized by a higher content of mitochondria, lipids, and myoglobin and a smaller diameter ${ }^{6}$. Previous studies have found that oxidative myofibers improved muscle growth and meat quality by affecting meat $\mathrm{pH}^{7-9}$, meat color ${ }^{10-12}$, and water-holding capacity ${ }^{8,13}$ in postnatal chickens. A higher content of oxidative myofiber makes the meat ruddy, fresh and juicy, which improves meat flavor, while a higher content of glycolytic myofiber makes the meat white and leads to reduced quality ${ }^{14}$. Therefore, how to regulate myofibers into the oxidative type is an important way to improve meat quality ${ }^{2,15-17}$.

Recent studies, most of which have been conducted among mice and pigs, have revealed the molecular mechanisms and possible signaling pathways involved in the proliferation of myoblasts and myofiber type. The expression level of PPAR in oxidative myofibers is significantly higher than that in glycolytic myofibers, and PPAR can induce the transformation of the oxidative type to glycolytic myofibers in mice ${ }^{18}$. Knocking out the AMPK $\beta 2$ gene could reduce the proportion of glycolytic myofibers in mice, and the AMPK $\beta 2$ gene plays an important role in the specificity and transformation of myofibers ${ }^{19}$. PPARGC1a is a major regulatory factor of mitochondrial synthesis and metabolism. In a mouse model, PPARGC1a cooperates with the MEF2 protein to activate the expression of oxidative fibro-related genes and is involved in the CaN signaling pathway as the target gene $^{20}$. Overexpression of the PPARGCla gene could promote glycolytic myofiber transformation to oxidative myofibers in pigs $^{21}$. Deletion of the MSTN gene induces muscle hypertrophy and increases the formation of glycolytic myofiber ${ }^{22}$. The role of noncoding RNAs (ncRNAs), especially miRNAs, in myofiber regulation has been extensively studied ${ }^{23}$. Several recent studies have also shown that long noncoding RNAs (lncRNAs) and circular RNAs (circRNAs) might also be involved in the regulation of myofiber type ${ }^{24-26}$. Currently, the in-depth exploration of lncRNAs and circRNAs involved in myofiber type is a new direction for meat quality improvement research. Shen et al..$^{27}$ used RNA-seq to discover a mass of candidate lncRNAs and circRNAs involved in porcine muscle physiological functions, which improved the understanding of muscle metabolism and development from an epigenetic perspective. Cai et al. ${ }^{28}$ found a novel muscle atrophy-associated lncRNA named SMUL that participates in the regulation of the transforming growth factor $\beta$ (TGF- $\beta$ )/SMAD pathway and further regulates myogenesis and muscle atrophy. Wang et al. ${ }^{29}$ found that lncRNA DLEU2 acts as a miR-181a sponge to regulate SEPP1 and inhibit skeletal muscle differentiation and regeneration. Gong et al..$^{30}$ found that a long noncoding RNA, LncMyoD, regulates skeletal muscle differentiation by blocking IMP2-mediated mRNA translation. Legnini et al. ${ }^{31}$ found that circ-ZNF609 precisely controlled myoblast proliferation. Ouyang et al. ${ }^{32}$ found that circRBFOX2 can sponge miR-206 and negatively regulate miR-206 expression, thus increasing CCND2 expression and promoting myoblast proliferation. Peng et al. ${ }^{33}$ found that circSNX29 acts as a miR-744 sponge and that increased Wnt5a and CaMKIId expression results in the activation of noncanonical Wnt pathways and myoblast differentiation. Currently, only a few lncRNAs and circRNAs have been identified for myofiber type determination in chickens.

Qingyuan partridge chicken, an important indigenous breed in China, is popular because of its high red muscle ratio, appealing meat color and flavor ${ }^{34,35}$. The breed's high oxidative metabolism leads to desirable muscle characteristics suitable for the study of muscle fiber types. In this study, the differential expression of lncRNAs and circRNAs between glycolytic muscle pectoralis major (PMM) and oxidative muscle sartorius (SART) of Chinese Qingyuan partridge chickens was analyzed to investigate the internal regulatory factors that regulate myofiber type.

\section{Results and discussion}

Phenotypic differences traits in oxidative and glycolytic myofiber. The luminance value $\left(\mathrm{L}^{\star}\right)$ of PMM was significantly higher than that of SART $(p<0.05)$, and the redness value $\left(\mathrm{a}^{\star}\right)$ of PMM was significantly lower than that of SART $(p<0.05$, Fig. 1A,B). The PMMs were all darkly stained and belonged to the glycolytic type, and the proportion of oxidative myofibers in sartorius muscle was $81.7 \%$, indicating that sartorius muscle was mainly composed of oxidative myofibers (Fig. 1C,D). The average diameter and cross-sectional area of the PMM were significantly higher than those of the SART $(p<0.05$, Fig. $1 \mathrm{E}-\mathrm{G})$, and the myofiber density was significantly higher than that of the SART $(p<0.05)$. The PMM and SART muscle could be used as ideal models to study myofiber types.

Screening of mRNAs, IncRNAs, and cricRNAs in oxidative and glycolytic myofibers. The RNASeq data from 8 cDNA libraries obtained 683,535,340 raw reads and 676,264,578 clean reads (Table 1). After discarding adaptor sequences and low-quality reads, approximately $78.50-85.48 \%$ of all reads were uniquely mapped to the chicken chromosomes, 0.59 to $1.04 \%$ were multiple mapped reads to the chicken chromosomes, and 79.27 to $86.13 \%$ of the clean reads were aligned against the Gallus gallus reference genome (Table 2). A total of 40,454 and 41,209 mRNAs were specifically expressed in the PMM and SART muscles, respectively, and 36,530 were expressed in both types of muscles. A total of 4517 and $4616 \operatorname{lncRNAs}$ were specifically expressed in PMM and SART muscles, respectively, and 3713 were expressed in both types of muscles. A total of 7697 and 6045 circRNAs were specifically expressed in PMM and SART muscles, respectively, and 3255 were expressed in both types of muscles (Fig. 2).

Identification of differentially expressed mRNAs, IncRNAs, and cricRNAs. In this study, replicates were highly correlated, with an average correlation coefficient of 0.92 (Fig. 3A). There were 3457 differentially expressed mRNAs (DE-mRNAs), including 2364 upregulated and 1093 downregulated mRNAs in the SART compared to the PMM. There were 365 differentially expressed lncRNAs (DE-lncRNAs), including 169 upregulated and 196 downregulated lncRNAs (Supplementary Table S1). There were 305 differentially expressed circRNAs (DE-circRNAs), including 111 upregulated and 194 downregulated circRNAs (Supplemen- 
A

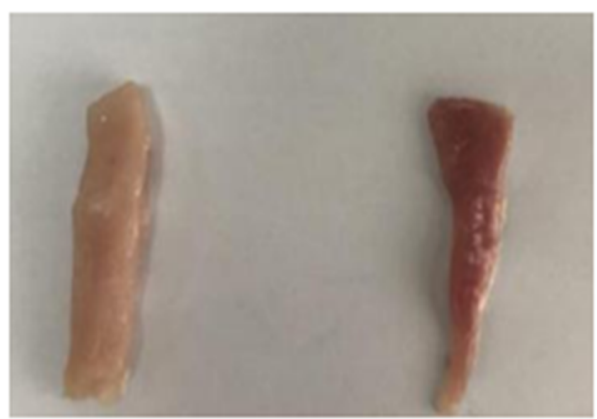

C
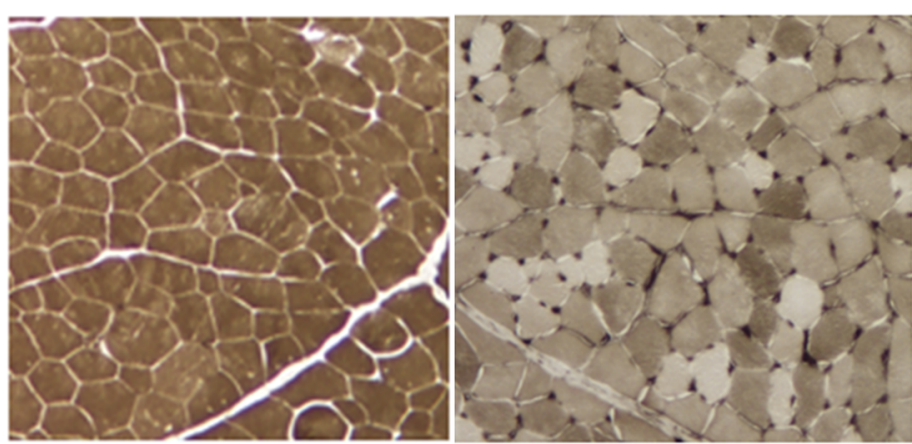

E

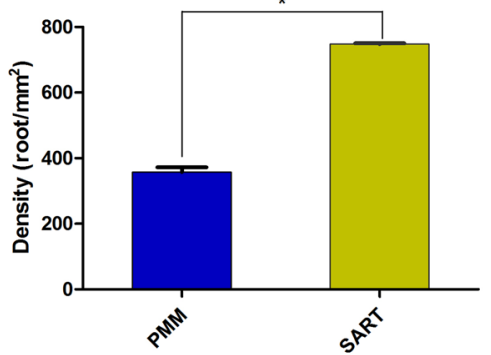

F

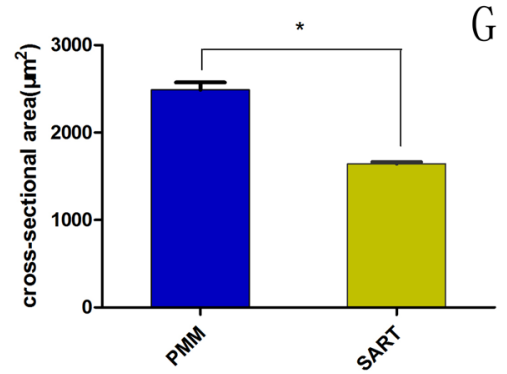

G
B

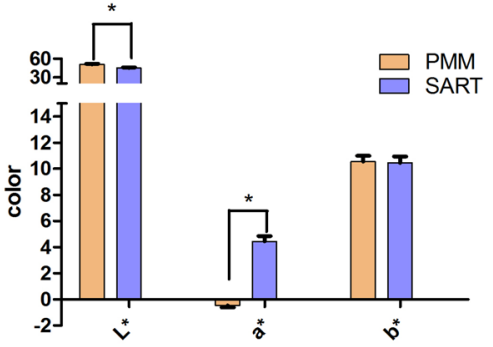

D
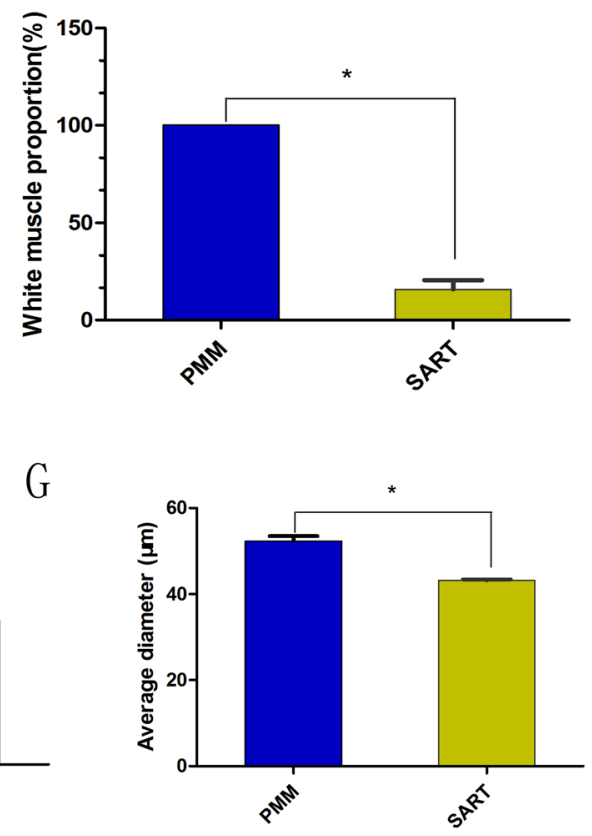

Figure 1. Different phenotypic indices between (pectoralis major) PMM and (sartorius major) SART muscles. (A) Fresh samples of PMM and SART, $n=15$. Left: PMM, right: SART; (B) The color of fresh samples of PMM and SART, $\mathrm{n}=15$; (C) ATPase alkaline incubation staining of muscles in PMM and SART, $\mathrm{n}=15$. Left: PMM, right: SART; (D) The myofiber type ratio (white/red) between PMM and SART, $n=15$; (E-G) The crosssectional area, density, and average diameter between PMM and SART, $n=15$. Data are means \pm SEM. Statistical significance was calculated by Student’s t-test. Significant difference levels: ${ }^{\star} p<0.05$.

\begin{tabular}{|l|l|l|l|l|l|l|l|}
\hline Sample & Raw reads num & Clean reads num & Raw data $(\mathbf{G b})$ & Clean data $(\mathbf{G b})$ & $\begin{array}{l}\text { Clean DataQ20 } \\
(\%)\end{array}$ & GC (\%) & rRNA reads (\%) \\
\hline PMM1 & $86,283,566$ & $85,474,788$ & 12.94 & 12.67 & 98.58 & 48.11 & 0.27 \\
\hline PMM2 & $80,733,792$ & $79,807,686$ & 12.11 & 11.82 & 98.61 & 48.36 & 0.34 \\
\hline PMM3 & $87,308,752$ & $86,360,624$ & 13.10 & 12.79 & 98.54 & 47.86 & 0.28 \\
\hline PMM4 & $81,087,082$ & $80,203,290$ & 12.16 & 11.88 & 98.76 & 49.04 & 0.53 \\
\hline SART1 & $80,773,526$ & $79,978,602$ & 12.12 & 11.86 & 98.66 & 46.74 & 0.62 \\
\hline SART2 & $94,089,360$ & $93,159,004$ & 14.11 & 13.82 & 98.60 & 46.99 & 0.93 \\
\hline SART3 & $82,498,530$ & $81,695,656$ & 12.37 & 12.11 & 98.68 & 46.85 & 0.72 \\
\hline SART4 & $90,760,732$ & $89,584,928$ & 13.61 & 13.26 & 98.58 & 46.93 & 0.73 \\
\hline
\end{tabular}

Table 1. RNA-seq date quality evaluation.

tary Table S2). Heatmaps generated from the expression of DE-mRNA, DE-lncRNA, and DE-circRNA were used to show the expression patterns of these RNAs between PMM and SART (Fig. 3B-D). These results proved the high reproducibility and reliability of transcriptome profiling performed in the present study. 


\begin{tabular}{|l|l|l|l|l|l|}
\hline Sample & Total reads & Unmapped reads (\%) & Unique mapped reads (\%) & Multiple mapped reads (\%) & Mapping ratio (\%) \\
\hline PMM1 & $85,243,206$ & 16.57 & 82.66 & 0.77 & 83.43 \\
\hline PMM2 & $79,534,030$ & 16.35 & 82.61 & 1.04 & 83.65 \\
\hline PMM3 & $86,120,530$ & 15.48 & 83.58 & 0.94 & 84.52 \\
\hline PMM4 & $79,780,262$ & 20.73 & 78.50 & 0.77 & 79.27 \\
\hline SART1 & $79,486,402$ & 14.71 & 84.61 & 0.68 & 85.29 \\
\hline SART2 & $92,293,538$ & 16.58 & 82.83 & 0.59 & 83.42 \\
\hline SART3 & $81,106,154$ & 13.87 & 85.48 & 0.66 & 86.13 \\
\hline SART4 & $88,934,220$ & 14.87 & 84.41 & 0.72 & 85.13 \\
\hline
\end{tabular}

Table 2. The results of mapped reads.
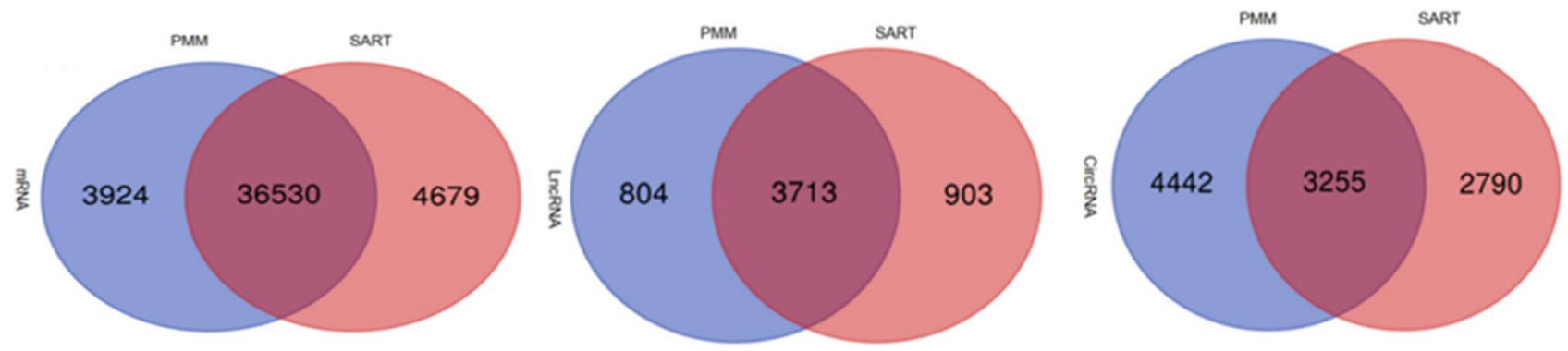

Figure 2. Venn diagrams represent all the numbers of expressed mRNAs, lncRNAs, and circRNAs between PMM and SART.

Functional enrichment analysis of neighboring target genes of differentially expressed IncRNAs. Recent studies suggested that lncRNAs may act in cis-regulation and affect the gene expression of their chromosomal neighborhood $10 \mathrm{~kb}$ upstream and downstream ${ }^{36,37}$. In this study, 49 significantly differentially expressed lncRNAs in PMM vs. SART were transcribed close to $(<10 \mathrm{~kb}) 70$ mRNAs. Gene Ontology (GO) analysis of the cis lncRNA target genes was performed to explore their functions (GO, https://www.omicshare. com/tools/home/report/goenrich.html) (Fig. 4A). There were 178 extremely significantly enriched GO terms $(p<0.05)$ in PMM vs. SART. Many of the significantly enriched biological processes were associated with muscle cell differentiation and myofiber regulation, such as striated muscle cell differentiation, regulation of cell proliferation, regulation of muscle cell differentiation, myoblast differentiation, and regulation of myoblast differentiation. Interestingly, T-box 3 (TBX3) was annotated in many myoblast differentiation-related GO terms. T-box3 (TBX3) could regulate the cell cycle, inhibit cell apoptosis and promote proliferation ${ }^{38,39}$ and was regulated by XR_003077811.1 in this study, which suggested that lncRNAs played important roles in regulating myofiber type.

To further understand the DE-lncRNA regulatory roles in myofiber type, Kyoto Encyclopedia of Genes and Genomes (KEGG, https://www.omicshare.com/tools/home/report/koenrich.html) pathway analysis was performed according to the cis-target genes (Fig. 4B). The MAPK signaling pathway was identified as a significantly enriched pathway for oxidative and glycolytic myofibers. Additionally, the MAPK family plays crucial roles in complex cellular processes, such as proliferation, differentiation, and development, by regulating the cell cycle and other cell proliferation-associated proteins ${ }^{40,41}$. Interestingly, only CALM2 was found to be differentially expressed in the MAPK signaling pathway and was regulated by XR_001465741.2. CALM2, as one subunit of $\mathrm{CaM}^{42}$, is a key redox-sensitive modulator of muscle physiology. Therefore, XR_001465741.2 was speculated to play a key role in myofiber growth.

Functional enrichment analysis of host genes targeted by differentially expressed circRNAs. Many studies have shown that circRNA expression can promote the transcription of host genes ${ }^{46-48}$. Therefore, to explore the mechanism of DE-circRNAs in myofibers, the host genes of DE-circRNA were used to perform a functional enrichment analysis (Fig. 5). There were 112 significantly enriched GO terms $(p<0.05)$ identified in the PMM and SART. The significantly overrepresented gene ontology (GO) terms were related to positive regulation of muscle hypertrophy, mitosis, positive regulation of developmental growth, and mononuclear cell proliferation (Fig. 5A). KEGG pathways, calcium signaling pathway, AMPK signaling pathway, FoxO signaling pathway, p53 signaling pathway, cellular senescence, etc., were closely related to myofiber development, resulting in the transformation of myofiber types (Fig. 5B). $\mathrm{Ca}^{2+}$ signaling pathways were implicated at every step of myogenesis ${ }^{49}$, which contributed to the transformation of glycolytic myofibers to oxidative myofiber type $e^{50}$. AMPK has three subunits: $\alpha, \beta$ and $\gamma ; \alpha$ is the catalytic subunit; and $\beta$ and $\gamma$ are the regulatory subunits. The distribution of the three subunits was related to muscle fiber type, and the $\beta 2$ subunit was highly expressed in skeletal muscle and mainly distributed in glycolytic muscle ${ }^{51}$. The AMPK signaling pathway regulates the differentiation directions of myoblasts and changes the types of myofiber ${ }^{52}$. The FoxO signaling pathway is regulated 
A

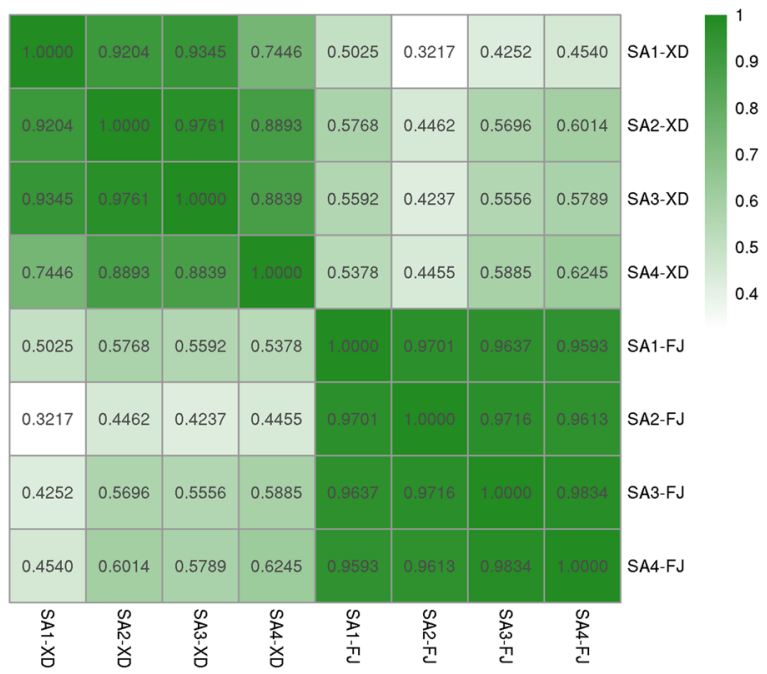

$\mathrm{C}$

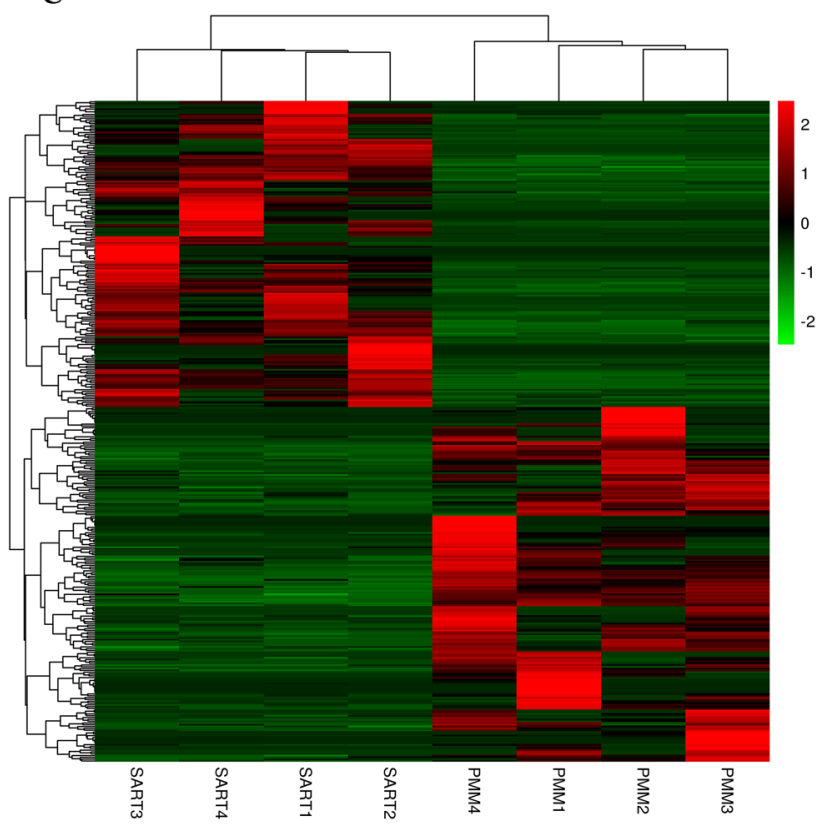

B

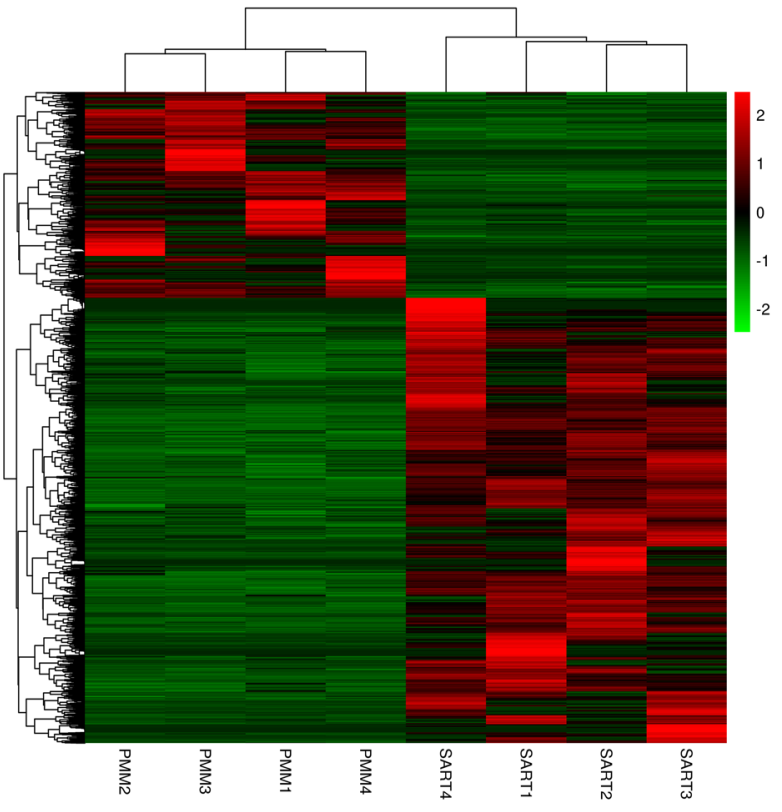

D

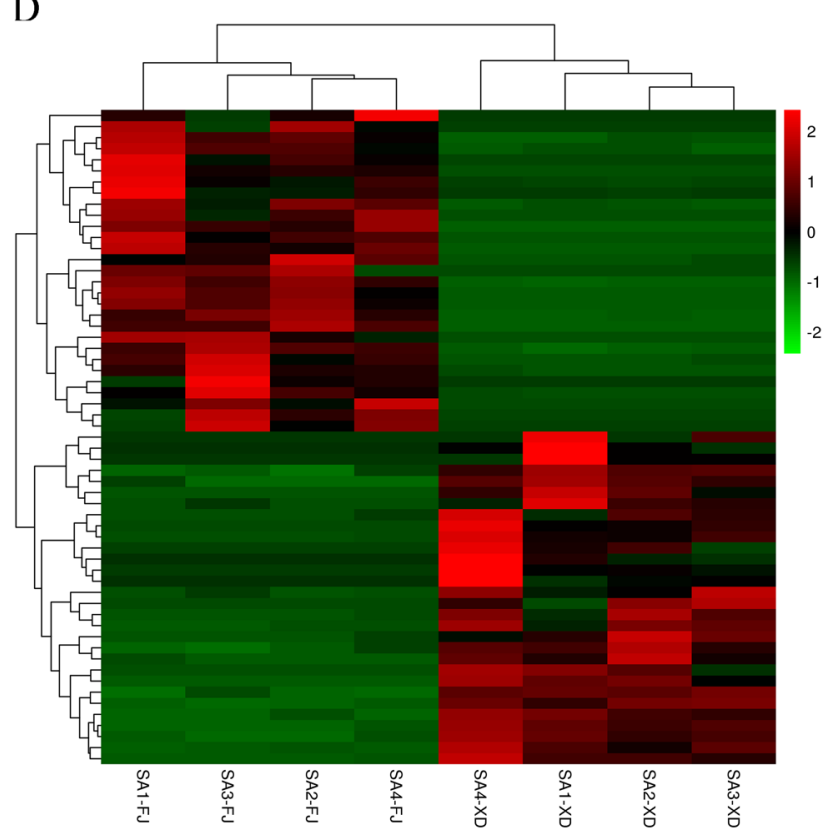

Figure 3. Heatmaps of sample correlation and differentially expressed RNAs between PMM and SART. (A) Sample consistency. Dark green indicates a high correlation. (B) Heatmap for DE-mRNA. (C) Heatmap for DE-lncRNA. (D) Heatmap for DE-circRNA.

by a variety of phosphorylated kinases and plays an important role in the proliferation and differentiation of myoblasts and the transformation of myofiber type ${ }^{53}$. p53-related genes promoted the cell cycle by upregulating p21 and enhancing muscle differentiation in MSTN knockout QM7 cells ${ }^{54}$. In this study, the host genes CAB39L, FOXO3, BMPR1B, SESN1, UBE2D1, PPP1CB, XPO1, NFATC1, BMPR1B, and PPP3CA of novel_circ_001395, novel_circ_003490, novel_circ_004266, novel_circ_003485, novel_circ_005184, novel_circ_003035, novel_ circ_002810, novel_circ_002121, novel_circ_004266, and novel_circ_004282 were involved in these pathways. These results suggest that these circRNAs might play important roles in regulating myofiber type.

Interestingly, differentially expressed novel_circ_004282 and novel_circ_002121 were transcribed from the PPP3CA and NFATC1 genes, respectively, which are CaN substrates involved in maintaining and regulating muscle phenotypes, promoting the growth of different types of myofibers, and maintaining muscle mass in the skeletal muscle of mammals after birth ${ }^{55-57}$. The CaN-NFAT pathway was the best candidate for an activity-dependent signaling pathway responsible for the maintenance of the oxidative gene program in adult oxidative muscles and the induction of this program in regenerating oxidative muscle ${ }^{58}$. These results suggested that novel_circ_004282 and novel_circ_002121 might directly regulate fiber type heterogeneity in muscles. 


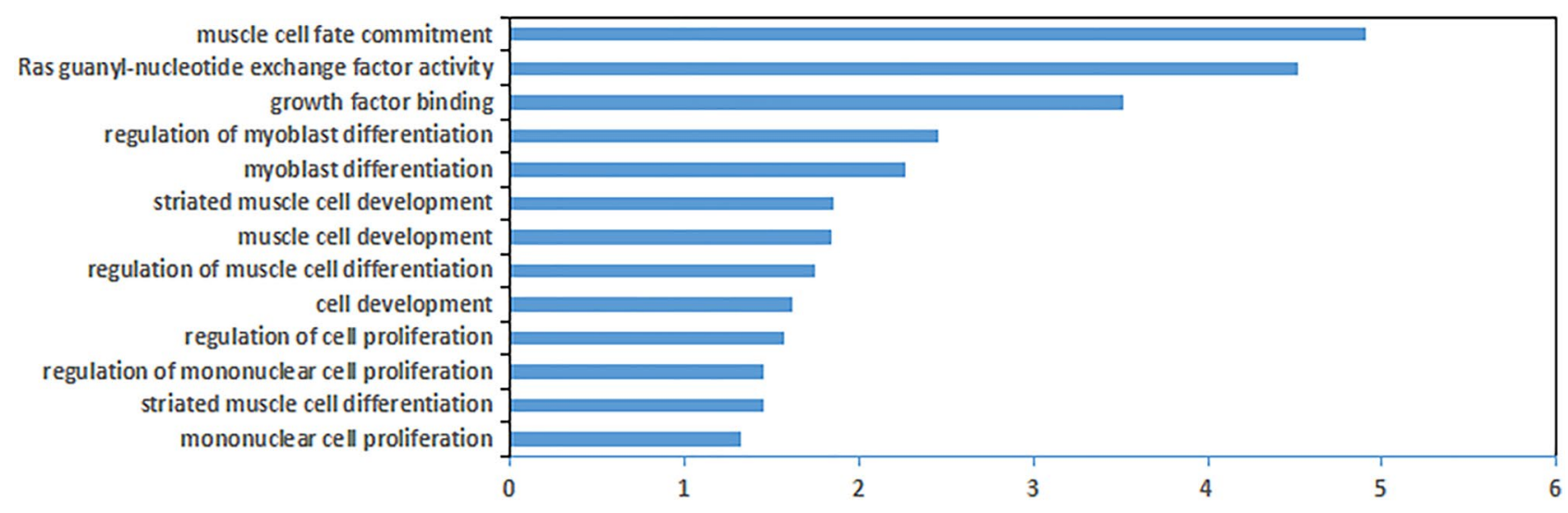

B

Top 20 of Pathway Enrichment

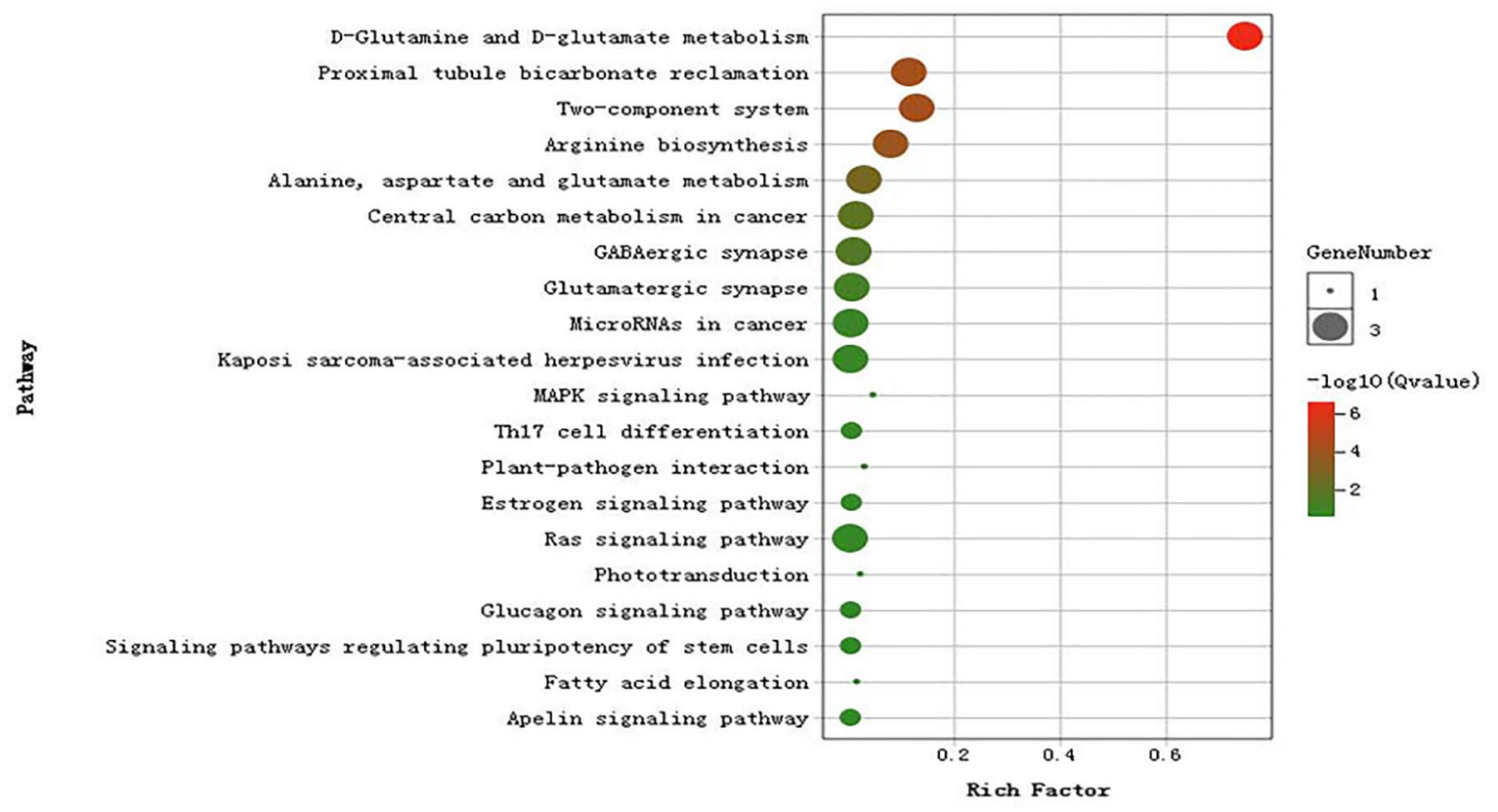

Figure 4. Functional enrichment analysis of neighboring target genes of DE-lncRNAs between PMM and SART. (A) Gene Ontology (GO) terms and (B) KEGG pathways enriched for neighboring target genes of DE-lncRNAs (cis-regulation) ${ }^{43-45}$.

LncRNA-mRNA interactions. To explore how lncRNAs interact with their target genes to regulate chicken myofibers and to identify key molecular players in the process, regulatory networks between DE-lncRNAs and their target DE genes were constructed. A total of 24 cis-regulatory interaction relationships were detected between 21 DE-lncRNAs and 24 DE-mRNAs (see Supplementary Fig. S1 online). DE-lncRNAs targeted 70 mRNAs, of which 24 mRNAs were differentially expressed and subjected to GO enrichment and KEGG pathway analysis. Six genes and 8 lncRNAs generated 8 interactions in GO terms (Fig. 6A), while 7 genes and 6 lncRNAs generated 7 interactions in KEGG pathways (Fig. 6B). The interaction networks containing TBX3, QKI, HSPA2, MYBPC1, CALM2, and PPARGC1A, which were regulated by XR_003077811.1, XR_001465942.2 or XR_003072304.1, XR_001470487.1 or XR_003077673.1, XR_001465741.2, XR_003074785.1, etc., IncRNAs, were all related to myofiber type. The interactions of XR_001466942.2-HSPA2 and XR_003072304.1-HSPA2 were both enriched in GO terms and KEGG pathways.

Over the past decades, QKI has important functions in neural progenitors, myelin formation, smooth muscle differentiation, and monocyte to macrophage differentiation ${ }^{59-61}$. QKI could promote the myoblast differentiation of $\mathrm{C} 2 \mathrm{C} 12$ cells in mice ${ }^{31}$. HSPA2 is abundantly expressed in skeletal muscle ${ }^{62}$ and is induced by exercise-associated oxidative stress ${ }^{63}$. It prevents apoptosis by interacting with apoptosis-inducing factors (AIFs) ${ }^{64}$. MYBPC1 is known to encode the oxidative myofiber isoform of the major myosin-binding proteins in muscles ${ }^{65-67}$ and acts as an adaptor to connect the ATP consumer (myosin) and the regenerator (MM-CK) ${ }^{68}$. The higher expression level of the MYBPC1 gene could result in more IMF deposition in skeletal muscle by controlling the energy 


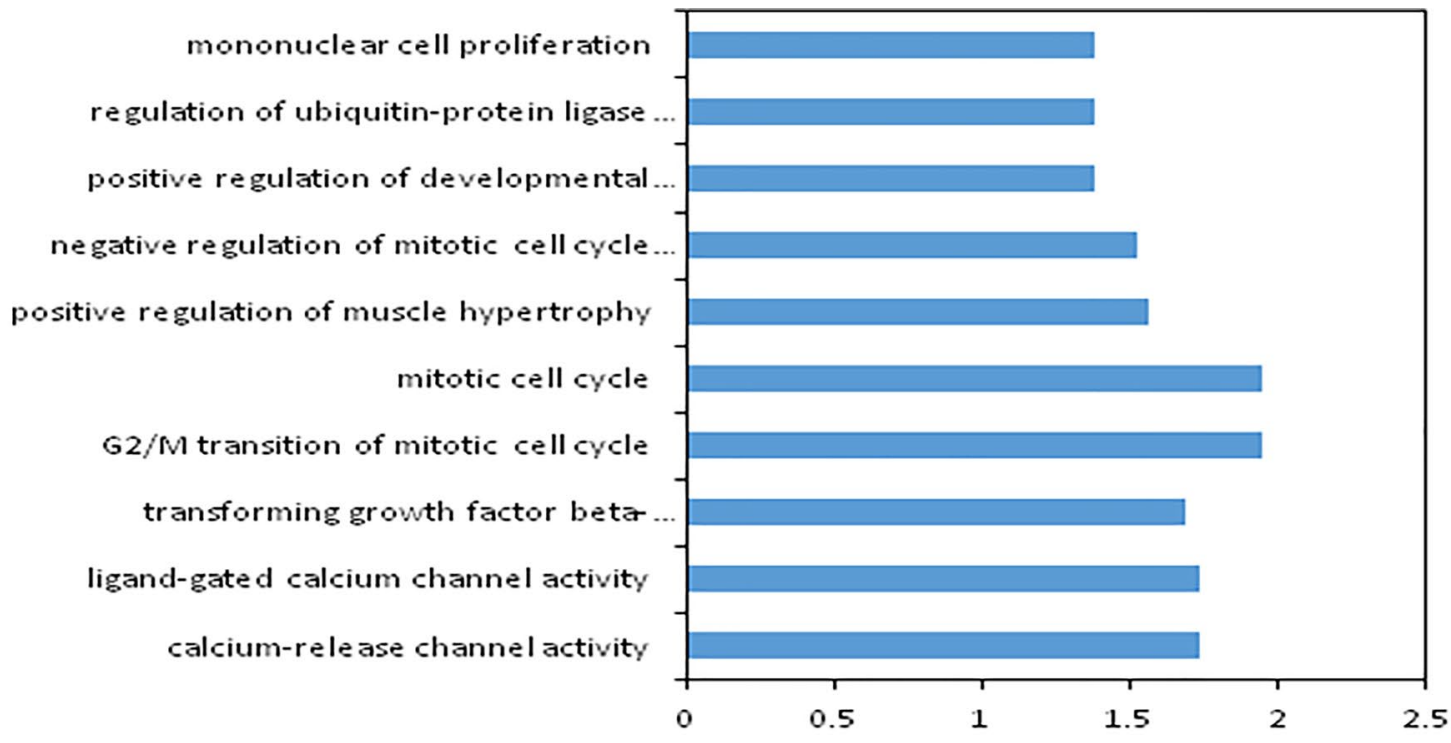

B

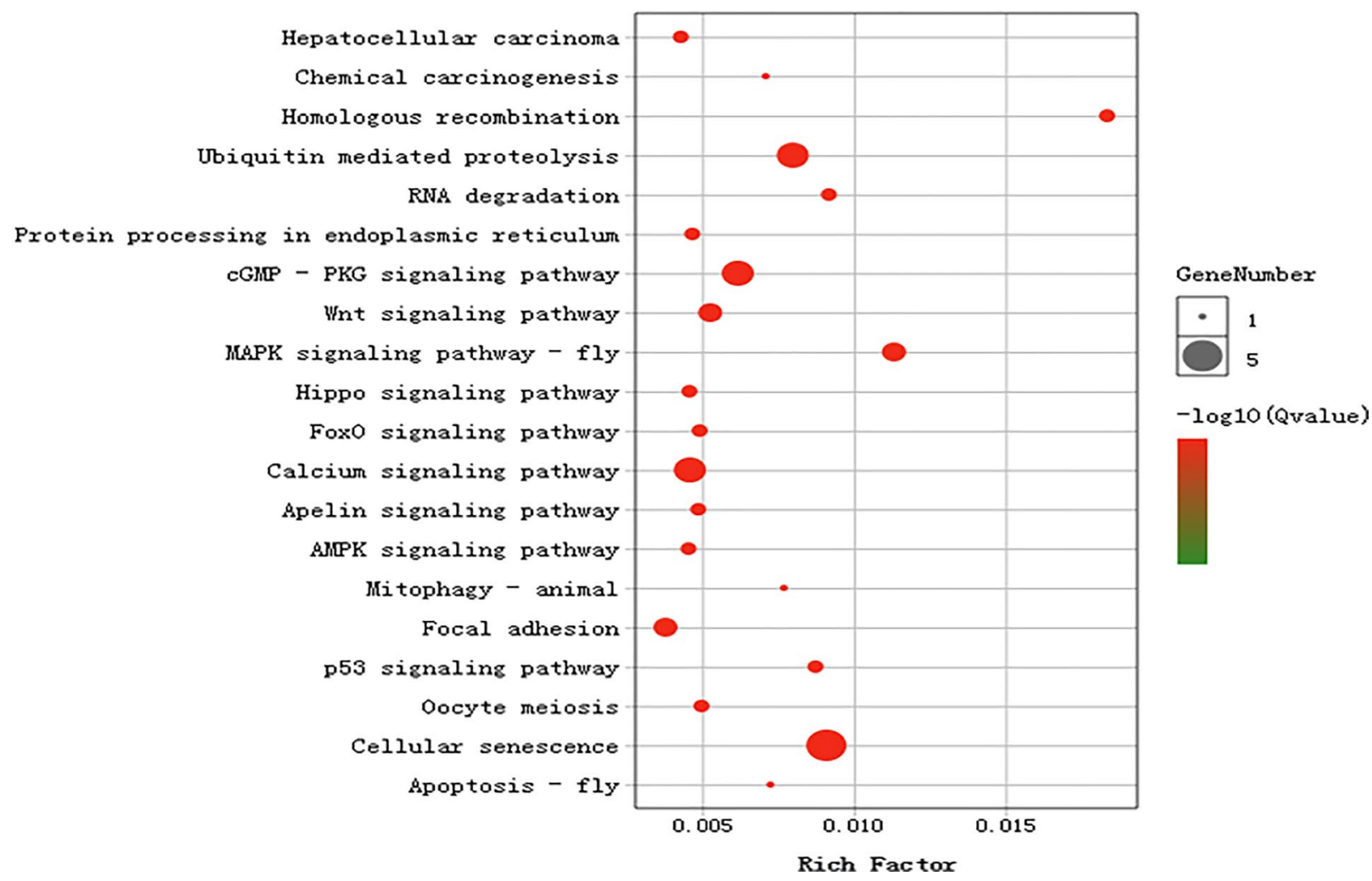

Figure 5. Functional enrichment analysis of differentially expressed host genes of circRNA between PMM and SART. (A) Gene Ontology (GO) terms and (B) KEGG pathways enriched for host genes of DE-circRNAs ${ }^{43-45}$.

metabolism and homeostasis of oxidative myofiber ${ }^{69}$. MYBPC1 could contribute to better meat quality of red muscle and was more highly expressed in oxidative myofibers than in glycolytic myofiber ${ }^{70}$. PPARGC1A is a coactivator of transcription involved in several aspects of skeletal muscle physiology, such as mitochondrial biogenesis, glucose utilization, fatty acid oxidation, thermogenesis, gluconeogenesis and insulin signaling ${ }^{71}$. Overexpression of PPARGC1A in mice reveals oxidative myofiber dominance ${ }^{20}$, while PPARGC1A knockout mice exhibit glycolytic myofiber dominance ${ }^{72}$. PPARGC1A has been shown to directly coactivate different myocyte enhancer factor 2 (Mef2) proteins involved in the induction and maintenance of muscle differentiation; this was considered critically important for switching among myofiber types or for myofiber transition to oxidative myofibers ${ }^{20}$. Previous research also confirmed that PPARGC1A was a key gene involved in chicken myofiber type $^{70}$. In this study, QKI, HSPA2, MYBPC1, and PPARGC1A were all highly expressed in SART. Therefore, these 
A
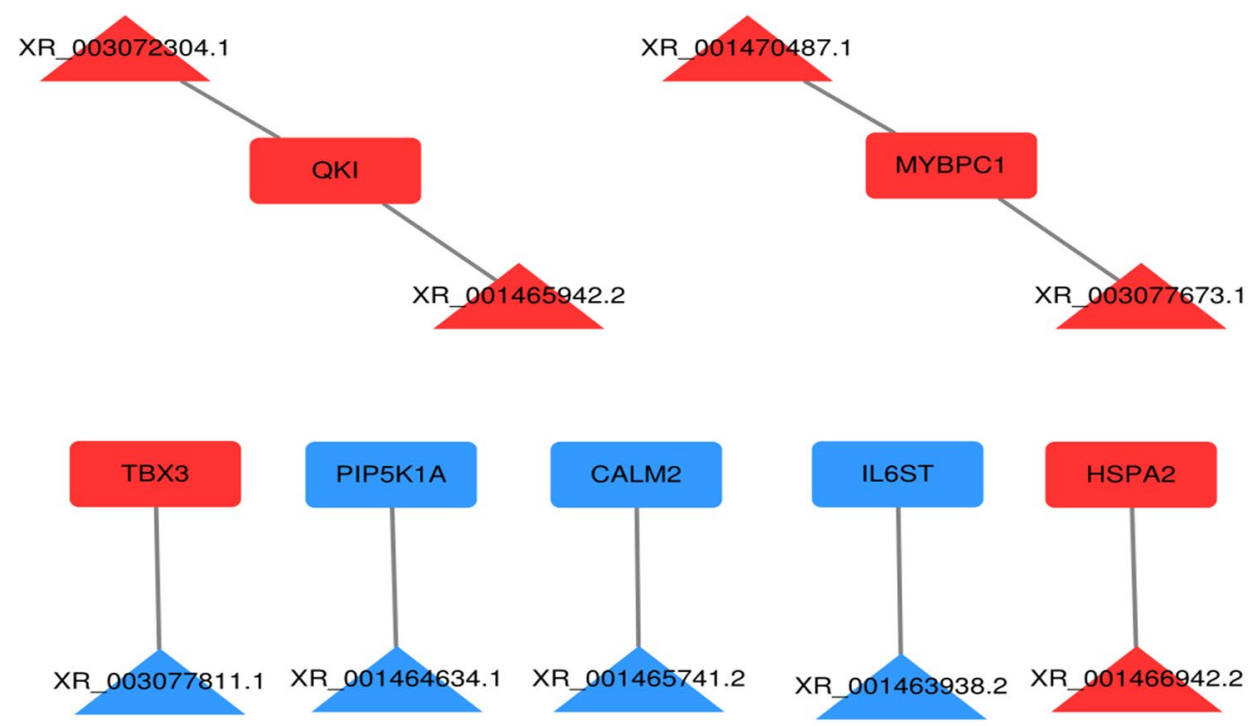

$\mathrm{B}$
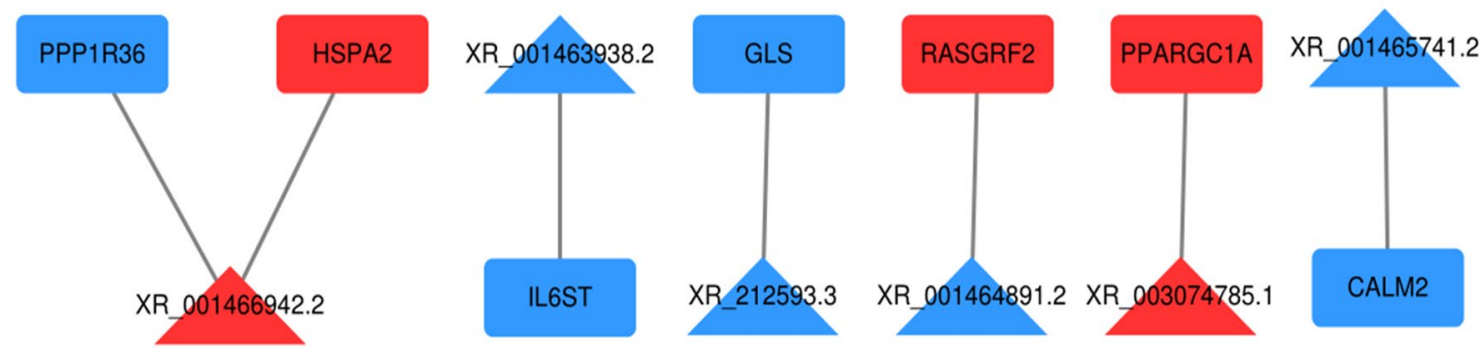

Figure 6. IncRNA-mRNA interactions for the selected cis-target DE genes. (A) lncRNA-mRNA interactions related to the GO terms. (B) lncRNA-mRNA interactions related to the KEGG signaling pathways. Genes are shown in rectangles, and lncRNAs are shown in triangles. RNA exhibiting upregulation is shown in red, whereas RNA exhibiting downregulation is shown in green.

results suggest that XR_003077811.1, XR_003072304.1, XR_001465942.2, XR_001465741.2, XR_001470487.1, XR_003077673.1 and XR_003074785.1 play important roles in regulating oxidative myofiber by TBX3, QKI, MYBPC1, CALM2, and PPARGC1A expression.

LncRNA-miRNA/circRNA-miRNA interaction networks in oxidative and glycolytic myofibers. In a previous study, the microRNA transcriptomes of SART and PMM of Chinese Qingyuan partridge chickens were compared ${ }^{4}$. Sixty-seven differentially expressed miRNAs were identified. To explore the lncRNAmiRNA/miRNA-miRNA interaction network, the target relationship between miRNAs and lncRNAs (Supplementary Table S3) and the target relationship between miRNAs and circRNAs during SART and PMM (Supplementary Table S4) were predicted. The role of miRNAs in myofiber type, such as gga-miR-499, gga-miR-1, gga-miR-196-5p, gga-miR-193a-3p, gga-miR-34a-5p, gga-miR-221-5p, and gga-miR-126-3p, has been extensively studied. MYH7B (oxidative myosin) is regulated by miR-499 and is responsible for muscle performance ${ }^{73}$. MiR-499-5p plays roles in the specification of myofiber identity, thereby promoting the NFATc1/MEF2C pathway and then activating a series of oxidative myofiber gene programs ${ }^{74}$. The miR-499/Fnip1/AMPK signaling pathway could serve as a mechanism to couple myofiber type and mitochondrial function ${ }^{75}$. MiR-1 plays an important role in myoblast differentiation, regeneration, angiogenesis regulation, proapoptosis, and oxidative stress control ${ }^{76}$. miR-1 expression could be regulated by IGF1 via the IGF1-AKT-FOXO3-miR-1 axis ${ }^{77}$. MiR-1 creates a positive regulatory feedback loop by targeting HDAC4 (histone deacetylase 4), a repressor of MEF2, which subsequently results in the upregulation of miR-1 ${ }^{78}$. MiR-196-5p was predicted to suppress CALM1 and MYLK4 expression, and CALM1 and MYLK4 were vital in regulating myofiber type ${ }^{79}$. Gga-miR-221, ggamiR-34a, and gga-miR-126 are involved in muscle cell differentiation and energy metabolisms ${ }^{80-82}$. Gga-miR193-3p inhibited PPARGC1A expression in chicken muscles. Gga-miR-129-3p has multiple target genes that are involved in the CaN/NFAT signaling pathway, suggesting its roles in chicken myofiber regulation through the CaN/NFAT signaling pathway ${ }^{4}$.

Therefore, gga-miR-499, gga-miR-1, gga-miR-196-5p, gga-miR-193a-3p, gga-miR-34a-5p, gga-miR-221-5p, and gga-miR-126-3p were selected as the targets to construct the miRNA-lncRNA interaction network diagram 
and miRNA-circRNA interaction network diagram (Fig. 7A,B). LncRNAs and circRNAs that regulate these miRNAs might regulate the type of myofiber.

LncRNA-miRNA-mRNA regulatory networks. To identify potential lncRNA-miRNA-mRNA regulatory networks in oxidative myofibers and glycolytic myofibers, lncRNA-miRNA-mRNA regulatory networks of DE-genes, DE-miRNAs, and DE-lncRNAs were constructed. Interestingly, the lncRNA-miRNA-mRNA regulatory networks containing lncRNAs (XR_003074785.1) and its cis-target gene (PPARGC1A) might combine with gga-miR-193-3p, lncRNA (XR_003074785.1) and PPARGC1A were upregulated, and gga-miR-193-3p was downregulated. Therefore, XR_003074785.1 might competitively combine with miR-193-3p and then inhibit its combination with the PPARGC1A 3' UTR, which might regulate the myofiber type in chickens (Fig. 8).

Validation of differentially expressed IncRNAs and circRNAs by qRT-PCR. To validate the differential expression results of lncRNAs and circRNAs, the relative expression of three randomly selected lncRNAs and three randomly selected circRNAs was quantified by qRT-PCR. In Fig. 9A,B, all selected DE-lncRNAs and DE-circRNAs showed concordant expression patterns between the RNA-seq and qRT-PCR results.

\section{Materials and methods}

Ethics statement. All animal experiments were performed in accordance with the protocol of the Animal Use Committee of the Chinese Ministry of Agriculture and were approved by the Animal Care and Use Committee at the Poultry Institute, Chinese Academy of Agricultural Science. All experiments were performed in accordance with relevant guidelines and regulations. The animals were euthanized according to the American Veterinary Medical Association (AVMA) Guidelines for the Euthanasia of Animals (2020). All efforts were made to minimize animal suffering. The reporting in the manuscript follows the recommendations in the ARRIVE guidelines and was in accordance with relevant guidelines and regulations.

Animal materials, tissue collection. A total of 15 female Qingyuan partridge chickens, provided by Guangdong Tiannong Food Ltd, Guangdong, China, with similar body weights were euthanized by stunning followed by exsanguination at 140 days of age (marketing age). Pectoralis major and sartorius major on the left side were sampled immediately after slaughter, one part of the samples was stored in liquid nitrogen for frozen sectioning, and the other part was stored in liquid nitrogen for RNA extraction. Pectoralis major and sartorius major on the right side were sampled and stored at $4{ }^{\circ} \mathrm{C}$ for meat color measurement.

Meat color measurement. After $24 \mathrm{~h}$ of storage, meat color was measured by a color difference meter (Shenzhen 3NH Technology CO., Ltd, Guangdong, China). The average of triplicate measurements was recorded, and the results were expressed as lightness $\left(L^{*}\right)$ and redness $\left(a^{*}\right)$ values.

Frozen section analysis. A $1 \times 1 \mathrm{~cm}^{2}$ section in the middle of the right SART and PMM was selected. Measurement of the myofiber characteristics, including density, cross-sectional area, average diameter, and myofiber ratios, was carried out using ATPase staining. Myosin ATPase staining was used to identify myofiber type and to measure myofiber size ${ }^{83,84}$. The lightly dyed fibers were type I, the darker dyed fibers were type IIB, and the middle of the dyeing was type IIA. Oxidative myofibers included type I and IIA myofibers, and glycolytic myofibers were type IIB myofibers.

RNA extraction. Total RNA was extracted by using TRIzol reagent (Invitrogen, CA, USA) following the manufacturer's protocol. The RNA quantity of each sample was examined using a NanoDrop ND-2000 spectrophotometer (Thermo Scientific, Waltham, MA, USA) at 260/280 nm (ratio > 2.0). The integrity of total RNA was analyzed with the Agilent Bioanalyzer 2100 and RNA 6000 Nano LabChip Kit (Agilent Technologies) with RNA integrity number $(\mathrm{RIN}) \geq 7$.

Transcriptome library construction and sequencing. A total of eight cDNA libraries were constructed with four PMMs and four SART muscle tissues. A total of $3 \mu \mathrm{g}$ RNA per sample was used as input material for RNA sample preparation. After the mRNAs and noncoding RNAs were enriched by removing rRNAs from the total RNA, the enriched RNAs were fragmented into short fragments and reverse transcribed into cDNAs. Buffer, dNTPs, RNase H, and DNA polymerase I were added to synthesize the second-strand cDNA. The resulting double-stranded cDNAs were ligated to adaptors after being end-repaired and A-tailed. Then, uracil-Nglycosylase (UNG) was used to digest the second-strand cDNAs. The digested products were size selected by agarose gel electrophoresis, PCR amplified, and sequenced by Gene Denovo Biotechnology Co. (Guangzhou, China) using Illumina HiSeq $4000^{85}$.

Identification of IncRNAs and circRNA. The Illumina sequencing raw reads were obtained by removing adapter sequences, reads with poly-N, and low-quality reads, in which the number of bases with a quality value $\mathrm{Q} \leq 20$ was $>50 \%$. All downstream analyses were based on high-quality clean data. The clean reads of each sample were mapped to the reference genome (Galgal 6.0) using TopHat (version 2.1.1) ${ }^{86}$. Reference genome and gene model annotation files were downloaded from a genome website (fttp://ftp.ensembl.org/pub/release-83/ fasta/gallus_gallus/dna/). The mapped reads from each library were assembled with Cufflinks (version 2.1.1) to construct and identify mRNA transcripts ${ }^{87}$. Transcript abundance was quantified by RSEM software ${ }^{88}$. 

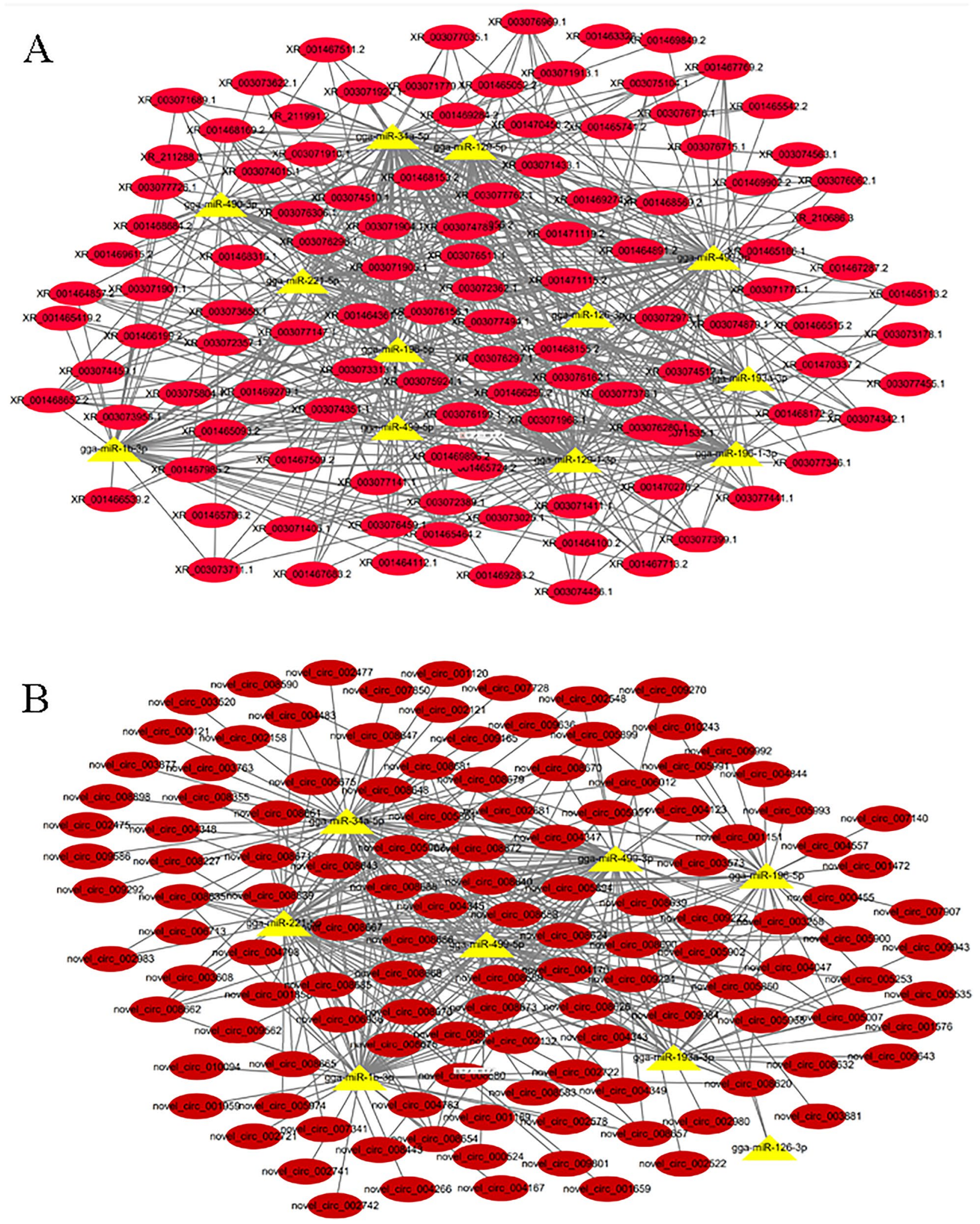

Figure 7. LncRNA-miRNA/circRNA-miRNA interaction network. (A) LncRNA-miRNA interaction network. LncRNA is shown in circular and red, miRNA is shown in the triangle, and yellow. (B) MircRNA-miRNA interaction network. CircRNA is shown in circular and red, miRNA is shown in the triangle, and yellow. 


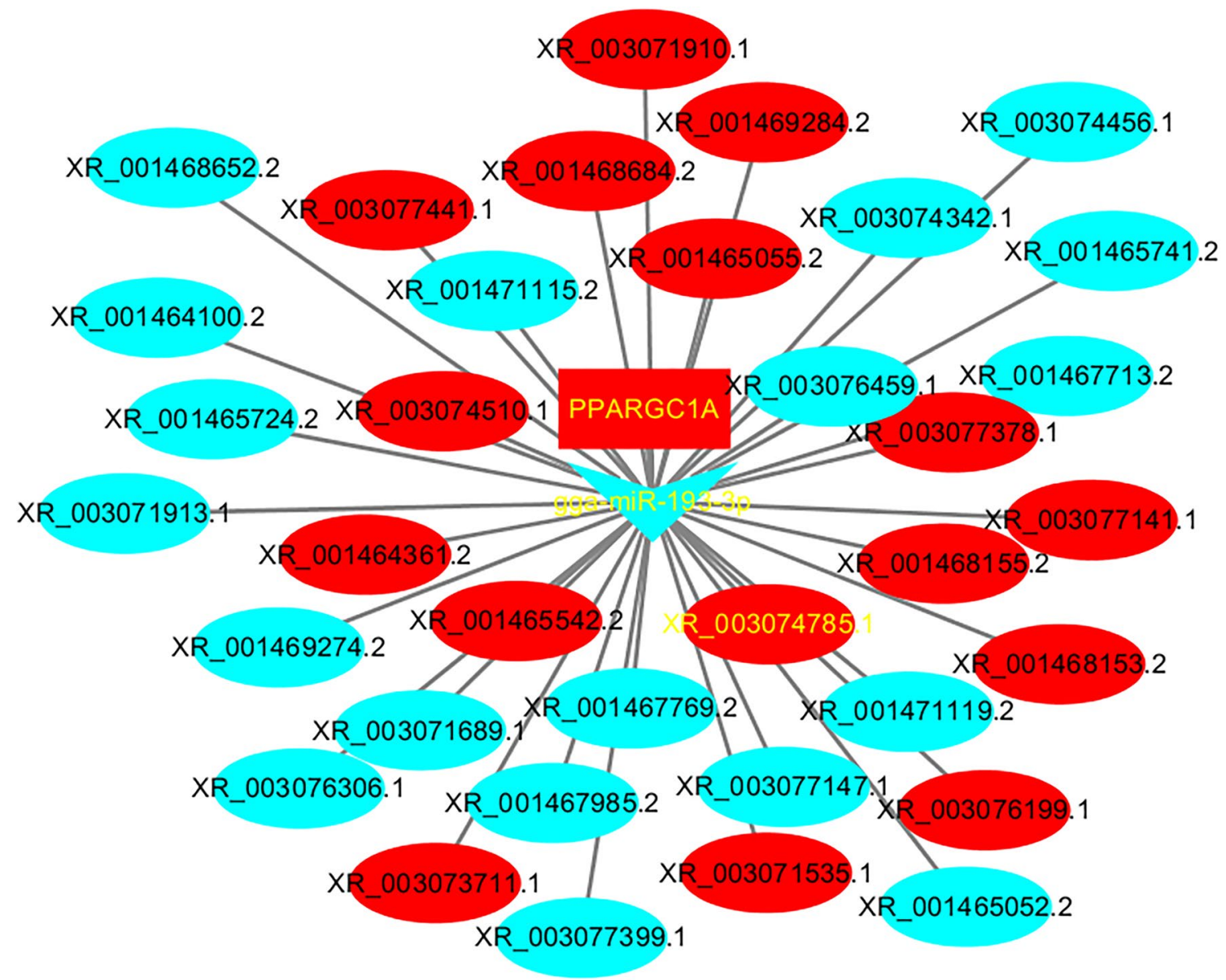

Figure 8. LncXR_003074785.1/miR-193-3p/PPARGC1A regulatory networks. LncRNA is shown in ellipse, miRNA is shown in the $\mathrm{V}$, the gene is shown in the rectangle. Red is upregulated and blue is downregulated.

A

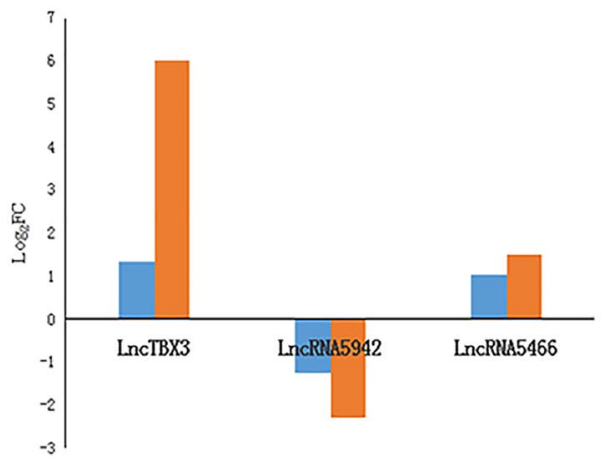

$\mathrm{B}$

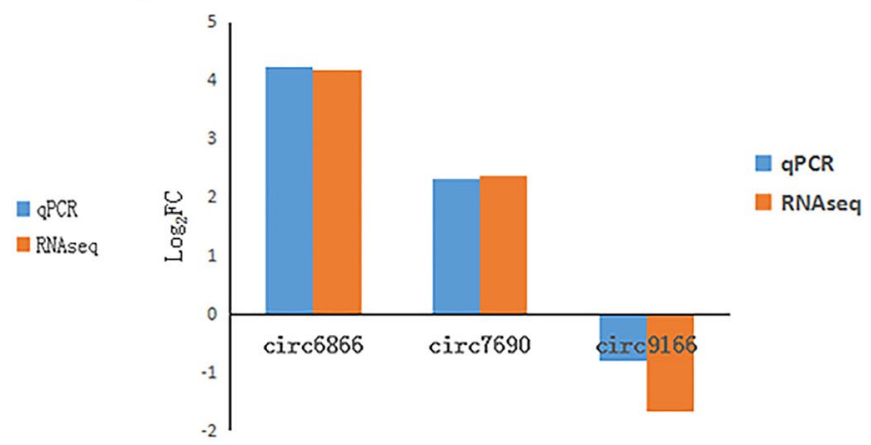

Figure 9. Using qRT-PCR to validate RNA-seq data. (A) RNA-seq and qRT-PCR data of the expression levels of lncRNAs, $n=3$. (B) RNA-seq and qRT-PCR data of the expression levels of circRNAs, $n=3$.

Next, new transcripts were screened based on the position of the assembled transcript on the reference genome and the following screening criteria: transcript length $\geq 200 \mathrm{bp}$ and the number of exons $\geq 2$ (plant sample $\geq 1$ ) to obtain known and new transcripts from the sample. Next, the Coding-Non-Coding Index $(\mathrm{CNCI})^{89}$ and Coding Potential Calculator $(\mathrm{CPC})^{90}$ were used to remove potential protein-coding transcripts. In this study, the resulting transcripts with no protein-coding potential in the two software analyses resulted in the lncRNA dataset.

In this study, find_circ software was used to identify circRNAs. Briefly, the unmapped back-spliced junction reads (default $20 \mathrm{bp}$ ) were used to extend the anchor sequences by find_circ software with default parameters ${ }^{91}$. In addition, identified circRNAs that were expressed in at least two samples were used for further analysis. 


\begin{tabular}{|c|c|c|}
\hline Gene symbol & Forward primer $\left(5^{\prime}-3^{\prime}\right)$ & Reverse primer $\left(5^{\prime}-3^{\prime}\right)$ \\
\hline LncRNA5942 & ATAGGGCAGGGGAGGCTAAATACC & GGTGTGTTGAGGCTCGTGATGG \\
\hline LncRNA5466 & CCACGGTTGACTTCAGCTCCTTC & TCCCGTAGTGTGTCTCCGATGTC \\
\hline LncTBX3 & TCCGCCAGTTCCGAGTGACC & AGACAAAGACTGTTCGCACCTTCC \\
\hline Circ6866 & TCGTGGCAGGAAGTCAGTGTC & TGAACATTCACCAAGGATCCGTGT \\
\hline Circ7690 & AACCAAAGCCTGGTCATACTCCT & GAGTGAGACTGCAGTCCATGGC \\
\hline Circ9166 & AACAGTGATGATTGGAGGAGAGCC & CAGAAAAGGAGTCTTTGGACAATGGT \\
\hline
\end{tabular}

Table 3. Primers for quantitative real-time PCR.

The expression levels of mRNAs, lncRNAs, and circRNAs were normalized using the fragments per kilobase of transcript per million mapped reads (FPKM) method. Differentially expressed mRNAs, lncRNAs, and cricRNAs were filtered by edgeR software with parameters of $\mathrm{p}$ value $<0.05$ and $\mid \log 2$ (fold change) $\mid \geq 1^{92}$.

Functional enrichment analysis. Differentially expressed lncRNAs were selected for cis-target gene predictions and were integrated with differentially expressed gene data to improve the veracity of target prediction. In the present study, DE-mRNAs located $\sim 10 \mathrm{~kb}$ upstream and downstream of DE-lncRNAs were classified as cis-acting target genes; then, their functional roles were predicted as follows. The host genes of DE-circRNA were used to perform a functional enrichment analysis.

All DE-mRNAs and target genes of DE-lncRNAs and DE-circRNAs were annotated and classified by Gene Ontology (GO) and Kyoto Encyclopedia of Genes and Genomes (KEGG) pathway analysis with OmicShare tools (HTTP ://www.omics hare.com/tools/ $)^{43-45}$. The results with a $p$ value $<0.05$ were considered significantly enriched. DE-mRNA and DE-lncRNAs were used to construct lncRNA-mRNA interaction networks by Cytoscape 3.5.1.

Construction of IncRNA-miRNA-mRNA network. The lncRNA-miRNA-mRNA network was constructed based on lncRNA-miRNA-mRNA theory as follows: (1) Expression correlation between mRNAmiRNA or lncRNA-miRNA was evaluated using the Spearman rank correlation coefficient (SCC). Pairs with SCC $<-0.7$ were selected as coexpressed negative lncRNA-miRNA pairs or mRNA-miRNA pairs, both mRNA and IncRNA were miRNA target genes, and all RNAs were differentially expressed. (2) The expression correlation between lncRNAs and mRNAs was evaluated using the Pearson correlation coefficient (PCC). Pairs with PCC $>0.9$ were selected as coexpressed lncRNA-mRNA pairs, and both mRNA and lncRNA in this pair were targeted and coexpressed negatively with a common miRNA. (3) A hypergeometric cumulative distribution function test was used to test whether the common miRNA sponges between the two genes were significant. As a result, only the gene pairs with a $p$ value less than 0.05 were selected ${ }^{93-95}$.

DE-miRNA, DE-mRNA, DE-lncRNAs, and DE-circRNAs were used to construct lncRNA-miRNA, circRNAmiRNA, and lncRNA-miRNA-mRNA interaction networks by Cytoscape 3.5.1.

Validation by qRT-PCR. To validate the differential expression results from sequencing, three lncRNAs and three circRNAs were selected for qRT-PCR. Total RNA for sequencing was reverse transcribed into cDNA using the PrimeScript RT reagent kit (TaKaRa, Dalian, China). Then, qRT-PCR was conducted using a KAPA SYBR Fast universal qPCR kit (Kapa Biosystems, USA). Glyceraldehyde-3-phosphate dehydrogenase (GAPDH) genes were used as the internal reference. All primers are shown in Table 3.

Statistical analysis. Comparisons of the two myofiber types were analyzed using an independent sample T-test procedure in SPSS (Version 20.0, SPSS, Inc., Chicago, IL, USA). Differences between PMM and SART muscle samples were considered statistically significant at $p<0.05$.

\section{Data availability}

The datasets supporting the results presented here are available in the Sequence Read Archive (SRA) repository under accession number PRJNA578179.

Received: 11 May 2021; Accepted: 7 October 2021

Published online: 21 October 2021

\section{References}

1. Matsakas, A. \& Patel, K. Skeletal muscle fibre plasticity in response to selected environmental and physiological stimuli. Histol. Histopathol. 24, 611-629. https://doi.org/10.14670/HH-24.611 (2009).

2. Choi, Y. M. \& Kim, B. C. Muscle fiber characteristics, myofibrillar protein isoforms, and meat quality. Livest. Sci. 122, 105-118 (2009).

3. Pette, D. \& Staron, R. S. Transitions of muscle fiber phenotypic profiles. Histochem. Cell Biol. 115, 359-372. https://doi.org/10. $1007 / \mathrm{s} 004180100268$ (2001).

4. Liu, Y. et al. miRNA-mRNA network regulation in the skeletal muscle fiber phenotype of chickens revealed by integrated analysis of miRNAome and transcriptome. Sci. Rep. 10, 10619. https://doi.org/10.1038/s41598-020-67482-9 (2020). 
5. Raj, S. et al. The relationship between the chemical composition of the carcass and the fatty acid composition of intramuscular fat and backfat of several pig breeds slaughtered at different weights. Meat Sci. 86, 324-330. https://doi.org/10.1016/j.meatsci.2010. $04.037(2010)$.

6. Holloszy, J. O. \& Coyle, E. F. Adaptations of skeletal muscle to endurance exercise and their metabolic consequences. J. Appl. Physiol. Respir. Environ. Exerc. Physiol. 56, 831-838. https://doi.org/10.1152/jappl.1984.56.4.831 (1984).

7. Choi, Y. M., Ryu, Y. C. \& Kim, B. C. Influence of myosin heavy- and light chain isoforms on early postmortem glycolytic rate and pork quality. Meat Sci. 76, 281-288. https://doi.org/10.1016/j.meatsci.2006.11.009 (2007).

8. Kim, G. D. et al. The influence of fiber size distribution of type IIB on carcass traits and meat quality in pigs. Meat Sci. 94, 267-273. https://doi.org/10.1016/j.meatsci.2013.02.001 (2013).

9. Ryu, Y. C. \& Kim, B. C. Comparison of histochemical characteristics in various pork groups categorized by postmortem metabolic rate and pork quality. J. Anim. Sci. 84, 894-901. https://doi.org/10.2527/2006.844894x (2006).

10. Kauffman, R. G. et al. Can pale, soft, exudative pork be prevented by postmortem sodium bicarbonate injection?. J. Anim. Sci. 76, 3010-3015. https://doi.org/10.2527/1998.76123010x (1998).

11. Kim, G. D., Jeong, J. Y., Hur, S. J., Yang, H. S. \& Joo, S. T. The relationship between meat color (CIE L* and a*), myoglobin content, and their influence on muscle fiber characteristics and pork quality. Korean J. Food Sci. Anim. Resour. 30, 626-633 (2010).

12. Renerre, M. Factors involved in the discoloration of beef meat. Int. J. Food Sci. Technol. 25, 613-630 (2010).

13. Larzul, C. et al. Phenotypic and genetic parameters for longissimus muscle fiber characteristics in relation to growth, carcass, and meat quality traits in large white pigs. J. Anim. Sci. 75, 3126-3137. https://doi.org/10.2527/1997.75123126x (1997).

14. Joo, S. T., Kim, G. D., Hwang, Y. H. \& Ryu, Y. C. Control of fresh meat quality through manipulation of muscle fiber characteristics. Meat Sci. 95, 828-836. https://doi.org/10.1016/j.meatsci.2013.04.044 (2013).

15. Lee, S. H., Joo, S. T. \& Ryu, Y. C. Skeletal muscle fiber type and myofibrillar proteins in relation to meat quality. Meat Sci. 86, 166-170. https://doi.org/10.1016/j.meatsci.2010.04.040 (2010).

16. Lefaucheur, L. A second look into fibre typing-relation to meat quality. Meat Sci. 84, 257-270. https://doi.org/10.1016/j.meatsci. 2009.05.004 (2010).

17. Shen, L. Y. et al. Effects of muscle fiber type on glycolytic potential and meat quality traits in different Tibetan pig muscles and their association with glycolysis-related gene expression. Genet. Mol. Res. GMR 14, 14366-14378. https://doi.org/10.4238/2015. November.13.22 (2015).

18. Schuler, M. et al. PGClalpha expression is controlled in skeletal muscles by PPARbeta, whose ablation results in fiber-type switching, obesity, and type 2 diabetes. Cell Metab. 4, 407-414. https://doi.org/10.1016/j.cmet.2006.10.003 (2006).

19. Steinberg, G. R. et al. Whole body deletion of AMP-activated protein kinase \{beta\}2 reduces muscle AMPK activity and exercise capacity. J. Biol. Inorg. Chem. 285, 37198-37209. https://doi.org/10.1074/jbc.M110.102434/2010.September.20 (2010).

20. Lin, J. et al. Transcriptional co-activator PGC-1 alpha drives the formation of slow-twitch muscle fibres. Nature 418, 797-801. https://doi.org/10.1038/nature00904 (2002).

21. Ying, F. et al. Muscle fiber-type conversion in the transgenic pigs with overexpression of PGC1a gene in muscle. Biochem. Biophys. Res. Commun. 480, 669-674. https://doi.org/10.1016/j.bbrc.2016.10.113 (2016).

22. Xuan, M. F. et al. Shift from slow- to fast-twitch muscle fibres in skeletal muscle of newborn heterozygous and homozygous myostatin-knockout piglets. Reprod. Fertil. Dev. 31, 1628-1636. https://doi.org/10.1071/RD19103 (2019).

23. Horak, M., Novak, J. \& Bienertova-Vasku, J. Muscle-specific microRNAs in skeletal muscle development. Dev. Biol. 410, 1-13. https://doi.org/10.1016/j.ydbio.2015.12.013 (2016).

24. Li, Y., Chen, X., Sun, H. \& Wang, H. Long non-coding RNAs in the regulation of skeletal myogenesis and muscle diseases. Cancer Lett. 417, 58-64. https://doi.org/10.1016/j.canlet.2017.12.015 (2018).

25. Wang, J. et al. Comprehensive analysis of differentially expressed mRNA, IncRNA and circRNA and their ceRNA networks in the longissimus dorsi muscle of two different pig breeds. Int. J. Mol. Sci. https://doi.org/10.3390/ijms20051107 (2019).

26. Zhang, P. et al. Circular RNA regulation of myogenesis. Cells https://doi.org/10.3390/cells8080885 (2019).

27. Shen, L. et al. Comprehensive analysis of lncRNAs and circRNAs reveals the metabolic specialization in oxidative and glycolytic skeletal muscles. Int. J. Mol. Sci. https://doi.org/10.3390/ijms20122855 (2019).

28. Cai, B. et al. Long noncoding RNA SMUL suppresses SMURF2 production-mediated muscle atrophy via nonsense-mediated mRNA decay. Mol. Ther. Nucleic Acids 23, 512-526. https://doi.org/10.1016/j.omtn.2020.12.003 (2021).

29. Wang, Y. et al. $\operatorname{lncRNA}$ DLEU2 acts as a miR-181 a sponge to regulate SEPP1 and inhibit skeletal muscle differentiation and regeneration. Aging 12, 24033-24056. https://doi.org/10.18632/aging.104095 (2020).

30. Gong, C. et al. A long non-coding RNA, LncMyoD, regulates skeletal muscle differentiation by blocking IMP2-mediated mRNA translation. Dev. Cell 34, 181-191. https://doi.org/10.1016/j.devcel.2015.05.009 (2015).

31. Legnini, I. et al. Circ-ZNF609 is a circular RNA that can be translated and functions in myogenesis. Mol. Cell 66, 22-37. https:// doi.org/10.1016/j.molcel.2017.02.017 (2017).

32. Ouyang, H. et al. Circular RNAs are abundant and dynamically expressed during embryonic muscle development in chickens. DNA Res. Int. J. Rapid Publ. Rep. Genes Genomes 25, 71-86. https://doi.org/10.1093/dnares/dsx039 (2018).

33. Peng, S. et al. Circular RNA SNX29 sponges miR-744 to regulate proliferation and differentiation of myoblasts by activating the Wnt5a/Ca(2+) signaling pathway. Mol. Ther. Nucleic Acids 16, 481-493. https://doi.org/10.1016/j.omtn.2019.03.009 (2019).

34. China National Commission of Animal Genetic Resources. Animal Genetic Resources in China: Poultry (China Agriculture Press, Beijing, 2011).

35. Shu, J. T. et al. Transcriptional co-activator PGC-1a gene is associated with chicken skeletal muscle fiber types. Genet. Mol. Res. GMR 13, 895-905. https://doi.org/10.4238/2014.February.14.19 (2014).

36. Ørom, U. A. et al. Long noncoding RNAs with enhancer-like function in human cells. Cell 143, 46-58. https://doi.org/10.1016/j. cell.2010.09.001 (2010).

37. Zhang, B. et al. The lncRNA Malat1 is dispensable for mouse development but its transcription plays a cis-regulatory role in the adult. Cell Rep. 2, 111-123. https://doi.org/10.1016/j.celrep.2012.06.003 (2012).

38. Chen, D. et al. Genetic analysis of the TBX3 gene promoter in ventricular septal defects. Gene 512, 185-188. https://doi.org/10. 1016/j.gene.2012.10.066 (2013).

39. Cao, M. et al. TBX3 deficiency accelerates apoptosis in cardiomyoblasts through regulation of P21 expression. Life Sci. 239, 117040. https://doi.org/10.1016/j.lfs.2019.117040 (2019).

40. Zhang, W. \& Liu, H. T. MAPK signal pathways in the regulation of cell proliferation in mammalian cells. Cell Res. 12, 9-18 (2002).

41. Li, Y. et al. LncRNAs and their regulatory networks in breast muscle tissue of Chinese Gushi chickens during late postnatal development. BMC Genom. 22, 44. https://doi.org/10.1186/s12864-020-07356-6 (2021).

42. Steil, A. W., Kailing, J. W., Armstrong, C. J., Walgenbach, D. G. \& Klein, J. C. The calmodulin redox sensor controls myogenesis. PLoS ONE 15, e0239047. https://doi.org/10.1371/journal.pone.0239047 (2020).

43. Kanehisa, M. \& Goto, S. KEGG: Kyoto encyclopedia of genes and genomes. Nucleic Acids Res. 28, 27-30. https://doi.org/10.1093/ nar/28.1.27 (2000)

44. Kanehisa, M. Toward understanding the origin and evolution of cellular organisms. Protein Sci. 28, 1947-1951. https://doi.org/ 10.1002/pro.3715 (2019)

45. Kanehisa, M. et al. KEGG: Integrating viruses and cellular organisms. Nucleic Acids Res. 49, D545-D551. https://doi.org/10.1093/ nar/gkaa970 (2021). 
46. Li, X. et al. Circular RNA circITGA7 inhibits colorectal cancer growth and metastasis by modulating the Ras pathway and upregulating transcription of its host gene ITGA7. J. Pathol. 246, 166-179. https://doi.org/10.1002/path.5125 (2018).

47. Siede, D. et al. Identification of circular RNAs with host gene-independent expression in human model systems for cardiac differentiation and disease. J. Mol. Cell. Cardiol. 109, 48-56. https://doi.org/10.1016/j.yjmcc.2017.06.015 (2017).

48. Song, Z. et al. Comprehensive analysis of the expression profile of circRNAs and their predicted protein-coding ability in the muscle of mdx mice. Funct. Integr. Genom. 20, 397-407. https://doi.org/10.1007/s10142-019-00724-w (2020).

49. Tu, M. K. et al. Calcium signaling in skeletal muscle development, maintenance and regeneration. Cell Calcium 59, 91-97. https:// doi.org/10.1016/j.ceca.2016.02.005 (2016).

50. Liu, Y. et al. Signaling pathways in activity-dependent fiber type plasticity in adult skeletal muscle. J. Muscle Res. Cell Motil. 26, 13-21. https://doi.org/10.1007/s10974-005-9002-0 (2005).

51. Dasgupta, B. et al. The AMPK $\beta 2$ subunit is required for energy homeostasis during metabolic stress. Mol. Cell. Biol. 32, $2837-2848$. https://doi.org/10.1128/MCB.05853-11 (2012).

52. Chalkiadaki, A. et al. Muscle-specific SIRT1 gain-of-function increases slow-twitch fibers and ameliorates pathophysiology in a mouse model of duchenne muscular dystrophy. PLoS Genet. 10, e1004490. https://doi.org/10.1371/journal.pgen.1004490 (2014).

53. Schachter, T. N. et al. Kinetics of nuclear-cytoplasmic translocation of Foxol and Foxo3A in adult skeletal muscle fibers. Am. J. Phys. Cell Physiol. 303, C977. https://doi.org/10.1152/ajpcell.00027 (2012).

54. Park, J. W. et al. Muscle differentiation induced by $\mathrm{p} 53$ signaling pathway-related genes in myostatin-knockout quail myoblasts. Mol. Biol. Rep. 47, 9531-9540. https://doi.org/10.1007/s11033-020-05935-0 (2020).

55. Oh, M. et al. Calcineurin is necessary for the maintenance but not embryonic development of slow muscle fibers. Mol. Cell. Biol. 25, 6629-6638 (2005).

56. Mitchell, P. O., Mills, S. T. \& Pavlath, G. K. Calcineurin differentially regulates maintenance and growth of phenotypically distinct muscles. Am. J. Physiol. Cell Physiol. 282, C984-C992. https://doi.org/10.1152/ajpcell.00483.2001 (2002).

57. Lomonosova, Y. N., Turtikova, O. V. \& Shenkman, B. S. Erratum to: Reduced expression of MyHC slow isoform in rat soleus during unloading is accompanied by alterations of endogenous inhibitors of calcineurin/NFAT signaling pathway. J. Muscle Res. Cell Motil. 37, 53 (2016).

58. Schiaffino, S., Sandri, M. \& Murgia, M. Activity-dependent signaling pathways controlling muscle diversity and plasticity. Physiology 22, 269-278. https://doi.org/10.1152/physiol.00009.2007 (2007).

59. de Bruin, R. G. et al. Quaking promotes monocyte differentiation into pro-atherogenic macrophages by controlling pre-mRNA splicing and gene expression. Nat. Commun. 7, 10846. https://doi.org/10.1038/ncomms10846 (2016).

60. van der Veer, E. P. et al. Quaking, an RNA-binding protein, is a critical regulator of vascular smooth muscle cell phenotype. Circ. Res. 113, 1065-1075. https://doi.org/10.1161/CIRCRESAHA.113.301302 (2013).

61. Wu, H. Y., Dawson, M. R., Reynolds, R. \& Hardy, R. J. Expression of QKI proteins and MAP1B identifies actively myelinating oligodendrocytes in adult rat brain. Mol. Cell. Neurosci. 17, 292-302. https://doi.org/10.1006/mcne.2000.0941 (2001).

62. Bonnycastle, L. L. et al. Cloning, sequencing, and mapping of the human chromosome 14 heat shock protein gene (HSPA2). Genomics 23, 85-93. https://doi.org/10.1006/geno.1994.1462 (1994).

63. Siu, P. M., Bryner, R. W., Martyn, J. K. \& Alway, S. E. Apoptotic adaptations from exercise training in skeletal and cardiac muscles. FASEB J. Off. Publ. Fed. Am. Soc. Exp. Biol. 18, 1150-1152. https://doi.org/10.1096/f.03-1291fje (2004).

64. Gurbuxani, S. et al. Heat shock protein 70 binding inhibits the nuclear import of apoptosis-inducing factor. Oncogene 22, 66696678. https://doi.org/10.1038/sj.onc.1206794 (2003).

65. Offer, G., Moos, C. \& Starr, R. A new protein of the thick filaments of vertebrate skeletal myofibrils. Extractions, purification and characterization. J. Mol. Biol. 74, 653-676. https://doi.org/10.1016/0022-2836(73)90055-7 (1973).

66. Pepe, F. A. \& Drucker, B. The myosin filament. III. C-protein. J. Mol. Biol. 99, 609-617. https://doi.org/10.1016/s0022-2836(75) 80175-6 (1975).

67. Sato, N. et al. A novel variant of cardiac myosin-binding protein-C that is unable to assemble into sarcomeres is expressed in the aged mouse atrium. Mol. Boil. Cell 14, 3180-3191. https://doi.org/10.1091/mbc.e02-10-0685 (2003).

68. Chen, Z. et al. Slow skeletal muscle myosin-binding protein-C (MyBPC1) mediates recruitment of muscle-type creatine kinase (CK) to myosin. Biochem. J. 436, 437-445. https://doi.org/10.1042/BJ20102007 (2011).

69. Tong, B. et al. Association of the expression level of the MYBPC1 gene in skeletal muscle with marbling trait in Japanese Black Beef Cattle. Ann. Anim. Sci. 15, 349-358 (2015).

70. Jingting, S. et al. Oxidative and glycolytic skeletal muscles show marked differences in gene expression profile in Chinese Qingyuan partridge chickens. PLoS ONE 12, e0183118. https://doi.org/10.1371/journal.pone.0183118 (2017).

71. Franks, P. W. et al. Common variation at PPARGC1A/B and change in body composition and metabolic traits following preventive interventions: The Diabetes Prevention Program. Diabetologia 57, 485-490. https://doi.org/10.1007/s00125-013-3133-4 (2014).

72. Handschin, C. et al. Skeletal muscle fiber-type switching, exercise intolerance, and myopathy in PGC-1alpha muscle-specific knock-out animals. J. Biol. Chem. 282, 30014-30021. https://doi.org/10.1074/jbc.M704817200 (2007).

73. van Rooij, E. et al. A family of microRNAs encoded by myosin genes governs myosin expression and muscle performance. Dev. Cell 17, 662-673. https://doi.org/10.1016/j.devcel.2009.10.013 (2009).

74. Xu, M. et al. MicroRNA-499-5p regulates skeletal myofiber specification via NFATc1/MEF2C pathway and Thrap1/MEF2C axis. Life Sci. 215, 236-245. https://doi.org/10.1016/j.lfs.2018.11.020 (2018).

75. Liu, J. et al. Coupling of mitochondrial function and skeletal muscle fiber type by a miR-499/Fnip1/AMPK circuit. EMBO Mol. Med. 8, 1212-1228. https://doi.org/10.15252/emmm.201606372 (2016).

76. Goljanek-Whysall, K. et al. MicroRNA regulation of the paired-box transcription factor Pax3 confers robustness to developmental timing of myogenesis. Proc. Natl. Acad. Sci. U.S.A. 108, 11936-11941. https://doi.org/10.1073/pnas.1105362108 (2011).

77. Duan, C., Ren, H. \& Gao, S. Insulin-like growth factors (IGFs), IGF receptors, and IGF-binding proteins: Roles in skeletal muscle growth and differentiation. Gen. Comp. Endocrinol. 167, 344-351. https://doi.org/10.1016/j.ygcen.2010.04.009 (2010).

78. Lu, L. et al. A Novel YY1-miR-1 regulatory circuit in skeletal myogenesis revealed by genome-wide prediction of YY1-miRNA network. PLoS ONE 7, e27596. https://doi.org/10.1371/journal.pone.0027596 (2012).

79. Mallinson, J., Meissner, J. \& Chang, K. C. Chapter 2. Calcineurin signaling and the slow oxidative skeletal muscle fiber type. Int. Rev. Cell Mol. Biol. 277, 67-101. https://doi.org/10.1016/S1937-6448(09)77002-9 (2009).

80. Liu, B. et al. miR-221 modulates skeletal muscle satellite cells proliferation and differentiation. In Vitro Cell. Dev. Biol. Anim. 54, 147-155. https://doi.org/10.1007/s11626-017-0210-x (2018).

81. Chen, Q. et al. miRNA-34a reduces neointima formation through inhibiting smooth muscle cell proliferation and migration. J. Mol. Cell. Cardiol. 89, 75-86. https://doi.org/10.1016/j.yjmcc.2015.10.017 (2015).

82. Wang, S. et al. The endothelial-specific microRNA miR-126 governs vascular integrity and angiogenesis. Dev. Cell 15, $261-271$. https://doi.org/10.1016/j.devcel.2008.07.002 (2008).

83. Brooke, M. H. \& Kaiser, K. K. Muscle fiber types: How many and what kind?. Arch. Neurol. 23, 369-379. https://doi.org/10.1001/ archneur.1970.00480280083010 (1970).

84. Guth, L. \& Samaha, F. J. Qualitative differences between actomyosin ATPase of slow and fast mammalian muscle. Exp. Neurol. 25, 138-152. https://doi.org/10.1016/0014-4886(69)90077-6 (1969).

85. Langmead, B. et al. Fast gapped-read alignment with Bowtie 2. Nat. Methods 9, 357-359. https://doi.org/10.1038/nmeth.1923 (2012). 
86. Kim, D. et al. TopHat2: Accurate alignment of transcriptomes in the presence of insertions, deletions and gene fusions. Genome Biol. 14, R36. https://doi.org/10.1186/gb-2013-14-4-r36 (2013).

87. Trapnell, C. et al. Differential gene and transcript expression analysis of RNA-seq experiments with TopHat and Cufflinks. Nat. Protoc. 7, 562-578. https://doi.org/10.1038/nprot.2012.016 (2012).

88. Li, B. \& Dewey, C. N. RSEM: Accurate transcript quantification from RNA-Seq data with or without a reference genome. $B M C$ Bioinform. 12, 323. https://doi.org/10.1186/1471-2105-12-323 (2011).

89. Sun, L. et al. Utilizing sequence intrinsic composition to classify protein-coding and long non-coding transcripts. Nucleic Acids Res. 41, e166. https://doi.org/10.1093/nar/gkt646 (2013).

90. Kong, L. et al. CPC: assess the protein-coding potential of transcripts using sequence features and support vector machine. Nucleic Acids Res. 35, W345-W349. https://doi.org/10.1093/nar/gkm391 (2007).

91. Memczak, S. et al. Circular RNAs are a large class of animal RNAs with regulatory potency. Nature 495, 333-338. https://doi.org/ 10.1038/nature11928 (2013).

92. Robinson, M. D., McCarthy, D. J. \& Smyth, G. K. edgeR: A Bioconductor package for differential expression analysis of digital gene expression data. Bioinformatics 26, 139-140. https://doi.org/10.1093/bioinformatics/btp616 (2010).

93. Thomson, D. W. et al. Endogenous microRNA sponges: Evidence and controversy. Nat. Rev. Genet. 17, 272-283. https://doi.org/ 10.1038/nrg.2016.20 (2016).

94. Salmena, L. et al. A ceRNA hypothesis: The Rosetta Stone of a hidden RNA language?. Cell 146, 353-358. https://doi.org/10.1016/j. cell.2011.07.014 (2011).

95. Miao, X. et al. Ovarian transcriptomic study reveals the differential regulation of miRNAs and lncRNAs related to fecundity in different sheep. Sci. Rep. 6, 35299. https://doi.org/10.1038/srep35299 (2016).

\section{Acknowledgements}

This research was funded by the China Agriculture Research Systems (CARS-41), Agricultural Independent Innovation Fund of Jiangsu Province (CX(20)3003), Jiangsu Modern Agriculture (broiler) Industrial Technology System Integration Innovation Center (JATS[2020]356), and Jiangsu Province Agricultural New Variety Creation Project (PZCZ201728).

\section{Author contributions}

X.J. conceived and designed the experiments. X.J., Y.S., Y.L., M.Z., Y.T., and G.J. performed the experiments and participated in data collection. X.J., J.Z. and X.C. analyzed the data. X.J. drafted the manuscript. Z.G. and J.S. revised the manuscript critically. All authors have read and agreed to the published version of the manuscript.

\section{Competing interests}

The authors declare no competing interests.

\section{Additional information}

Supplementary Information The online version contains supplementary material available at https://doi.org/ 10.1038/s41598-021-00176-y.

Correspondence and requests for materials should be addressed to Z.G. or J.S.

Reprints and permissions information is available at www.nature.com/reprints.

Publisher's note Springer Nature remains neutral with regard to jurisdictional claims in published maps and institutional affiliations.

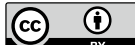

Open Access This article is licensed under a Creative Commons Attribution 4.0 International License, which permits use, sharing, adaptation, distribution and reproduction in any medium or format, as long as you give appropriate credit to the original author(s) and the source, provide a link to the Creative Commons licence, and indicate if changes were made. The images or other third party material in this article are included in the article's Creative Commons licence, unless indicated otherwise in a credit line to the material. If material is not included in the article's Creative Commons licence and your intended use is not permitted by statutory regulation or exceeds the permitted use, you will need to obtain permission directly from the copyright holder. To view a copy of this licence, visit http://creativecommons.org/licenses/by/4.0/.

(C) The Author(s) 2021 